\title{
D-serine has antidepressant effects in mice through suppression of the BDNF signaling pathway and regulation of synaptic plasticity in the nucleus accumbens
}

\author{
Zhenzhen Chen ${ }^{1}$, Zhenyu Tang ${ }^{1}$, Ke Zou ${ }^{2}$, Zhihong Huang ${ }^{1}$, Liuer Liu ${ }^{1}$, Yuan-Jian Yang ${ }^{3}$, \\ and Wei Wang ${ }^{1}$ \\ ${ }^{1}$ Nanchang University Second Affiliated Hospital \\ ${ }^{2}$ Jiangxi Mental Hospital of Nangchang University \\ ${ }^{3}$ Jiangxi Mental Hospital/Affiliated Mental Hospital of Nanchang University
}

March 27, 2021

\begin{abstract}
Background and Purpose: D-serine is a crucial endogenous co-agonist of NMDARs in the central nervous system and can affect the function of the BDNF system, which plays an essential role in modulating synaptic plasticity. The aim of the current investigation was to systematically evaluate the role and mechanisms of D-serine in depressive behavior in NAc. Experimental Approach: D-Serine concentration in the CSDS model in NAc was measured by HPLC. The antidepressant-like effects of Dserine were identified by the FST and TST in control mice, and then assessed in the CSDS model. We applied social interaction and sucrose preference tests to identify the susceptibility of CSDS model. Western blotting was further performed to assess the changes of BDNF signaling cascade in NAc after CSDS and D-serine treatment. The BDNF signaling inhibitor (K252a) was also used to clarify the antidepressant mechanism of D-serine. Moreover, effects of D-serine on synaptic plasticity in NAc were investigated by electrophysiological methods. Key Results: D-serine injections into the NAc exhibited antidepressant effects in the FST, TST and CSDS model. Next, D-serine down-regulated the BDNF signaling pathway in NAc during the CSDS procedure. Moreover, K252a enhanced the antidepressant effects of D-serine. We also found that D-serine was essential for NMDARs-LTD. Conclusion and Implications: Our results provide the first evidence that D-serine exerts antidepressant effects in mice mediated through restraining the BDNF signaling pathway and regulating synaptic plasticity in NAc, which indicates that D-serine may be an effective therapeutic agent for depression. KEYWORDS D-serine, depression, NAc, BDNF, CSDS, LTD
\end{abstract}

RESEARCH PAPER

D-serine has antidepressant effects in mice through suppression of the BDNF signaling pathway and regulation of synaptic plasticity in the nucleus accumbens

Zhenzhen Chen ${ }^{1}$,Zhenyu Tang ${ }^{1}$, Ke Zou ${ }^{2}$, Zhihong Huang ${ }^{1}$, Liuer Liu ${ }^{1}$,Yuanjian Yang ${ }^{2}$,Wei Wang ${ }^{1}$

${ }^{1}$ Department of Neurology, The Second Affiliated Hospital of Nanchang University, Nanchang 330006, Jiangxi, China

2Jiangxi Mental Hospital of Nangchang University, 43 Shangfang Road, Nanchang, Jiangxi 330029, PR China

\section{Correspondence}

Yuanjian Yang, Jiangxi Mental Hospital of Nangchang University, 43 Shangfang Road, Nanchang, Jiangxi 330029, PR China 
Wei Wang, Department of Neurology, The Second Affiliated Hospital of Nanchang University, Nanchang 330006, Jiangxi, China.

E-mail: yuanjimyang@yeah.net (Y. Yang),wuhansy@126.com (W. Wang).

Zhenzhen Chen and Zhenyu Tang contributed equally to this work.

\section{Funding information}

National Natural Science Funds of China, Grant/Award Numbers: 81760256 and 81960256; Academic and technical leaders of major disciplines Foundation, Grant/Award Numbers: 20204BCJL22049; Natural Science Foundation, Grant/Award Numbers: 20192BAB205038

\section{Abbreviations}

NMDARs, N-methyl-D-aspartate receptors; NAc, nucleus accumbens; CSDS, chronic social defeat stress; HPLC, high performance liquid chromatography; BDNF, brain-derived neurotrophic factor; TrkB, tyrosine receptor kinase B; CREB, cAMP response element-binding protein; FST, forced swimming test; TST, tail suspension test; LTD, long-term depression; fEPSPs, field excitatory postsynaptic potential; LFS, lowfrequency stimulation.

\section{Abstract}

Background and Purpose: D-serine is a crucial endogenous co-agonist of N-methyl-D-aspartate receptors (NMDARs) in the central nervous system and can affect the function of the brain derived neurotrophic factor (BDNF) system, which plays an essential role in modulating synaptic plasticity. The aim of the current investigation was to systematically evaluate the role and mechanisms of D-serine in depressive behavior in nucleus accumbens (NAc).

Experimental Approach: D-Serine concentration in the chronic social defeat stress (CSDS) model in NAc was measured by high-performance liquid chromatography (HPLC). With fluoxetine as the positive control, the antidepressant-like effects of D-serine were identified by the forced swim test (FST) and tail suspension test (TST) in control mice, and then assessed in the CSDS model. We applied behavioral tests of social interaction and sucrose preference tests to identify the susceptibility of CSDS model. Western blotting was further performed to assess the changes of BDNF signaling cascade in NAc after CSDS and D-serine treatment. The BDNF signaling inhibitor (K252a) was also used to clarify the antidepressant-like mechanism of D-serine. Moreover, effects of D-serine on synaptic plasticity in NAc were investigated by electrophysiological methods.

Key Results: The concentration of D-serine was decreased in depression susceptible mice in NAc. D-serine injections into the nucleus accumbens exhibited antidepressant-like effects in the FST and TST without affecting the locomotor activity of mice. D-serine was also effective in the CSDS model of depression. Moreover, D-serine down-regulated the BDNF signaling pathway in NAc during the CSDS procedure. Furthermore, BDNF signaling inhibitor (K252a) enhanced the antidepressant effects of D-serine. We also found that D-serine was essential for NMDARs-dependent long-term depression (LTD).

Conclusion and Implications: Our results provide the first evidence that D-serine exerts antidepressantlike effects in mice mediated through restraining the BDNF signaling pathway and regulating synaptic plasticity in NAc, which indicates that D-serine may be an effective therapeutic agent for depression.

\section{KEYWORDS}

D-serine, depression, nucleus accumbens (NAc), brain-derived neurotrophic factor (BDNF), chronic social defeat stress (CSDS), LTD

\section{Introduction}

Depression is a mental disorder characterized by notable, persistent and life-threatening mood disorders, and it is projected to be a major reason for disability worldwide by 2030 according to published reports from 
the World Health Organization(He et al., 2019). It causes huge social and economic consequences(Jin, Cui, Zhao, Fan, \& Li, 2019). In the past few decades, there has not yet been a highly efficient drug for treating depression(D. Li et al., 2020). Therefore, it is very necessary to develop more reliable antidepressants with fewer side effects.

BDNF is distributed throughout the central nervous system(Arora, Sharma, \& Singh, 2020). Currently, a leading hypothesis of depression suggests that the BDNF signaling pathway is closely implicated in the pathophysiology of depression(Caroleo et al., 2019). Phosphorylation and activation of cAMP response element binding protein (CREB) induced by BDNF binding to tyrosine kinase B (TrkB) receptor on the cell membrane(Bai, Zhang, Zhou, Li, \& Bai, 2019). CREB is a crucial transcription factor in the brain which regulates the biosynthesis of numerous pro-survival proteins, including BDNF(Alhusaini et al., 2020). We have testified that the level of activity of the BDNF signaling pathway is increased in the NAc of CSDS mice, whereas chronic antidepressant treatment could reverse these pathological changes(B. Jiang et al., 2014). These researches manifest that suppression of the NAc BDNF signaling pathway could offer a novel method to the therapy of depression.

BDNF plays an essential role in modulating synaptic plasticity(Kowiański et al., 2018; Tomassoni-Ardori et al., 2019). Development of depression has been ascribed to disfunction of the reward pathway, in which the NAc plays a key role(Lorsch et al., 2018). Chronic stress has been recorded to cause drastic neurochemical changes in the NAc, leading to depressive phenotypes(Shirayama \& Chaki, 2006). Our research has confirmed that synaptic adaptability in the NAc is the key to mediate depression(B Jiang et al., 2013; M. Li et al., 2018). NMDAR- LTD in the NAc as prime regulators in the remodeling of excitatory synapses and lingering psychomotor springiness in response to psychostimulant. D-serine is a crucial endogenous co-agonist of NMDARs in the central nervous system and has been recorded to influence the function of the BDNF system. It was found that CSDS exposure destabilized the D-serine in the NAc(Wook Koo et al., 2016). This destabilization constitutes a continued molecular adaptability of excitatory synapses to chronic stress, leading to the corresponding development of behavioral plasticity. Accumulating evidence reveals the impairment of NMDAR-dependent LTD in the NAc of animal models of depression(Belujon \& Grace, 2014).

In short, BDNF system and synaptic plasticity in NAc are closely related to depression, and D-serine affects the function of BDNF system and synaptic plasticity in NAc. However, there is no detailed study on the role of BDNF system and synaptic plasticity in NAc in D-serine-mediated antidepressant mechanism. The aim of the current investigation was to systematically evaluate the role of D-serine on depression behaviors considered to be mediated by the BDNF system and in LTD in the NAc.

\section{What is already known}

D-serine is a crucial endogenous co-agonist of NMDARs in the central nervous system and plays an essential role in modulating synaptic plasticity.

D-serine can affect the function of the BDNF system.

\section{What this study adds}

D-serine exerts antidepressant-like effects in mice mediated through restraining the BDNF signaling pathway and regulating synaptic plasticity in NAc.

\section{What is the clinical significance}

D-serine may be developed into a novel and effective therapeutic agent for depression.

\section{Methods}

\subsection{Animals}

Adult male C57BL/6J mice (9 weeks old) and male CD1 mice (50 weeks old) were obtained from the Experimental Animal Center of Medical College, Nanchang University. Before being used, mice were housed five per cage under standard conditions (12 hours light/dark cycle; lights on from 7:00 to 19:00; $23 \pm 1^{\circ} \mathrm{C}$ 
ambient temperature; $55 \pm 10 \%$ relative humidity; bedding replacement twice a week) for 1 week with free access to food and water. Each experimental group consisted of 20 mice and behavioral experiments were performed during the light phase. A total of 900 mice were used in these experiments. For animal sacrifice, all mice were first anaesthetized using $0.5 \%$ pentobarbital sodium $\left(10 \mathrm{~mL} \mathrm{~kg}^{-1}\right)$ and then killed by cervical dislocation. All animal care and experimental protocols were conducted in compliance with the Guide for the Care and Use of Laboratory Animals (8th edition, Institute of Laboratory Animal Resources on Life Sciences, National Research Council, National Academy of Sciences, Washington DC) and the guidelines of the Animal Care and Use Committees of Nanchang University, China. The animals were left undisturbed for at least one week between testing when used in multiple models. The home cages were brought into the test room at least 30 min before each experiment.

\subsection{Drugs}

Fluoxetine was purchased from Sigma (St. Louis, Missouri), dissolved in 0.9\% saline. The K252a was obtained from Sigma and dissolved in DMSO to $25 \mathrm{mM}$ and in ethanol to $5 \mathrm{mM}$. D-serine was provided by Sigma and dissolved in $0.9 \%$ saline.

\subsection{Treatment schedules}

The repeated drug therapy of CSDS mice was implemented once daily at 9:30 a.m.-10:30 a.m. in time of the last 14 days. The doses chosen were based on the behavioral consequences and previous records $(\mathrm{Xu}$, Wang, Zhao, Gao, \& Cui, 2017). All these compounds were stereotactic injected into the bilateral nucleus accumbens (NAc). Control mice were given the corresponding vehicle, also in the parallel volume.

\subsection{Intranucleus accumbens (NAc) infusions}

Briefly, C57BL/6J mice were anaesthetized with $0.5 \%$ pentobarbital sodium solution $\left(50 \mathrm{mg} \cdot \mathrm{kg}^{-1}\right.$, i.p. $)$ and fixed in a stereotaxic apparatus (Kopf, Tujunga, CA), with the anaesthetizing effects evaluated by muscle relaxation, slow corneal reflex, and no skin pinch reaction. The scalp was cut, and the skull was exposed using $75 \%$ alcohol and $1 \% \mathrm{H}_{2} \mathrm{O}_{2}$. The cannulas were implanted into the bilateral NAc according to bregma: anteroposterior, $+1.5 \mathrm{~mm}$; mediolateral, $+1.0 \mathrm{~mm}$; dorsoventral, $-4.5 \mathrm{~mm}$ (B Jiang et al., 2013). The cannula was cemented in place, and the incision was sutured. The animals were allowed to recover for at least 5 days after surgery. Osmotic minipumps were designed to deliver bilateral microinjection of different amounts of D-serine (2ug/perside ; $5 \mathrm{ug} /$ perside), K252a (5 ug/perside), fluoxetine (5 ug/perside), or saline (0.9\%) into the NAc daily for 14 days. Each osmotic minipump was attached to a brain infusion cannula. A total volume of $1.0 \mathrm{uL}$ was infused into each side over $15 \mathrm{~min}$, and the injection syringe was maintained in place for an additional 5 minutes to limit reflux along the injection track. In some experiments, a nonspecific tyrosine kinase inhibitor, K252a (5ug/perside), was co-infused bilaterally with D-serine, K252a was infused bilaterally 20 minutes before infusion of D-serine.

\subsection{High performance liquid chromatography analysis (HPLC)}

Tissue samples were weighed after extraction and homocysteine as an internal standard was added. After that the samples were homogenized in 10 volumes of $5 \%$ trichloroacetic acid (TCA) and the homogenates were centrifuged at $180006 \mathrm{~g}$ at $4 \mathrm{uC}$ for $30 \mathrm{~min}$. To remove TCA, the supernatants were washed three times with water-saturated diethylether. The resultant samples were used for HPLC analysis. Amino acid enantiomers were separated by HPLC using a carbon 18 reverse-phase column $(250 \mathrm{~mm}$ ) (Knauer, Advanced scientific instruments, Germany) with fluorimetric detection after derivatization with N-isobutyryl-L-cysteine and Ophthalaldehyde (Tomassoni-Ardori et al.). The Nisobutyryl-L-cysteine/OPA derivatives were immediately applied to the HPLC system (Knauer, Advanced scientific instruments, Germany). Mobile phase was $8 \%$ $\mathrm{MeCN}$ in $0.1 \mathrm{M}$ sodium acetate buffer ( $\mathrm{pH}$ 6.0). Amino acids were separated isocratically for 37 minutes and then the column was eluted with $50 \% \mathrm{H}_{2} \mathrm{O} / 50 \% \mathrm{MeCN}$ for 5 minutes. After that, the column was rebalanced for 15 minutes under the initial conditions. The flow-rate was $0.125 \mathrm{ml} \mathrm{min}^{-1}$. Fluorescence detection of each amino acid derivative was carried out at $443 \mathrm{~nm}$ with excitation at $344 \mathrm{~nm}$. The absolute D-serine levels referring to the internal standard were calculated and data were related to the wet weight of the initial tissue 
samples.

\subsection{Chronic social defeat stress}

The social defeat stress model of depression was performed according to our previously reported methods(X. Jiang, Lin, Cheng, \& Wang, 2020). Adult male C57BL/6J mice were the subjects, and CD1 retired breeders were the aggressors. Each C57BL/6J mice was exposed to a different aggressive CD1 mice each day for 10 minutes over a total of 14 days. After the 10 minutes of contact, the defeated C57BL/6J mice were separated from the CD1 aggressors for the next 24 hours by plastic dividers containing holes, where they were exposed to chronic defeat stress in the form of threaten. Undefeated control mice were handled daily. Twenty-four hours after the last stress, defeated C57BL/6J mice were subjected to the social interaction test and sorted into either susceptible or unsusceptible phenotype based on interaction scores. All the CSDS-unsusceptible mice were removed. Then the CSDS-susceptible mice were housed individually and received daily injections of tested drugs or vehicle for another 14 days. After that, various behavioral tests were performed.

\subsection{Forced swim test}

The FST test was carried out according to previously reported methods with slight modification(Zhong et al., 2020), and separate groups of C57BL/6J mice were used for this test. Briefly, 30 min after a single injection, mice were individually placed in a glass cylinder (height $45 \mathrm{~cm}$, diameter $20 \mathrm{~cm}$ ) filled with $25^{\circ} \mathrm{C}$ water to a depth of $15 \mathrm{~cm}$ for $6 \mathrm{~min}$. All mice were forced to swim for $6 \mathrm{~min}$, and the duration of immobility during the last 4 min interval of the test was recorded by an investigator blind to the study. The water was replaced after each trial. A mouse was judged to be immobile when it remained floating in the water without struggling and only making movements necessary to keep the nose above the water. The observers were unaware of the treatment of mice.

\subsection{Tail suspension test}

The FST is a widely used test for assessing potential antidepressant-like medications. The TST test was measured according to the methods described previously(Zhong et al., 2020), and separate groups of C57BL/6J mice were used for this test. Briefly, $30 \mathrm{~min}$ after a single injection, the test $\mathrm{C} 57 \mathrm{BL} / 6 \mathrm{~J}$ mice were suspended $60 \mathrm{~cm}$ above the floor for $6 \mathrm{~min}$ by adhesive tape placed approximately $1 \mathrm{~cm}$ from the tip of the tail. The duration of immobility was scored between the 2nd and 6th minute for $4 \mathrm{~min}$ by an investigator blind to the treatment. Mice were considered immobile only when they hung passively and were completely motionless, and any mice that did climb their tails were removed from the experimental analysis. The observers were unaware of the treatment of the mice. Means and SEM were calculated for each group.

\subsection{Open field test}

Spontaneous locomotor activity of experimental C57BL/6J mice was studied in the open field paradigm over a 5-min period(Zhi, Jin, Pan, Zhang, \& Liu, 2019), and separate groups of animals were used for this test. The open field was divided into two areas, a peripheral area and a square center. C57BL/6J mice were individually placed in the central of a wooden box (height $40 \mathrm{~cm}$, width $100 \mathrm{~cm}$, length $100 \mathrm{~cm}$ ). The test room was illuminated with a red bulb $(50 \mathrm{~W})$ on the ceiling dimly. For open field observations, the animals were placed in the central sector 30 minutes after a single injection. The computer software (EthoVision; Noldus) calculated the velocity of movement, the distance of traveling, and the time spent in the center of the open field. For each group, the mean value and SEM was calculated. These parameters are thought to reflect locomotor activity and fear or anxiety, respectively. The open field apparatus was thoroughly cleaned between each trial.

\subsection{Social interaction experiments}

Adult male C57BL/6J mice were the subjects, and CD1 retired breeders were the aggressors. The day after the last injection (day 29), the social interaction test was performed. The social interaction test comprises two trials of 5 minutes for each. In the first trial ('target absent'), each mouse was placed into an open-field box and allowed to explore a plastic enclosure placed within the predefined interaction zone. In the second trial 
('target present'), each mouse was returned to the open-field arena containing a plastic enclosure now holding an unfamiliar CD1 mouse. The duration in the interaction zone was obtained using Ethovision XT (Noldus, USA) software (in seconds). The open-field apparatus was cleaned after each trial to remove olfactory cues.

\subsection{Sucrose Preference Test (SPT)}

This test lasted for 4 days and was carried out according to previously reported methods with some modifications(Li-Wen et al., 2020). On the first 2 days, the C57BL/6J mice were free to drink two bottles containing pure water and $1 \%$ sucrose solution, respectively. And the position of two bottles was changed every $6 \mathrm{~h}$ to prevent potential location preference of drinking. On the 3rd day, both the food and two bottles were deprived for 24 hours. On the 4th days, the test lasted for 6 hours, with the position of the bottles interchanged and the two bottles weighed before and after the test period. Sucrose preference was calculated as the percentage of sucrose solution ingested relative to the total amount of liquid intake. Drug treatments were not given during the testing days.

\subsection{Western blotting experiments}

The experiment was processed according to previous reports with some modifications(Taylor \& Posch, 2014). Animals were killed the day after behavioral studies. To extract the total proteins, bilateral NAc were immediately dissected and homogenized in $10 \mathrm{mM}$ Tris $\mathrm{HCl} \mathrm{pH} \mathrm{8,} 150 \mathrm{mM} \mathrm{NaCl}, 5 \mathrm{mM}$ EDTA, $1 \%$ NP-40, $0.5 \%$ sodium deoxycholate, and 0.1\% SDS containing Complete Mini protease inhibitor cocktail (Snyder \& Kim), and then kept on ice for $30 \mathrm{~min}$ (Ntoukas et al., 2020). The lysates were centrifuged and supernatants were collected. Then, the samples were mixed with loading buffer and boiled for 5 min. Protein concentration was estimated using the BCA Protein Assay (Pierce). After denaturation, equal amounts of protein samples (30 mg) were separated by 10\% SDS/PAGE gel and then transferred to nitrocellulose membranes (Bio-Rad, Hercules, CA, USA). After being blocked with 10\% non-fat dried milk powder/Tris-buffered saline Tween-20 (TBST) for $1 \mathrm{~h}$, membranes were incubated overnight at 4with primary antibodies to BDNF (1:500; Abcam, UK), TrkB (1:1000; Abcam, UK), phospho-TrkB-tyr515 (p-TrkB;1:500; Abcam, UK), cAMP response element-binding protein CREB (1:1000; Abcam, UK), phospho-CREB-Ser133 (pCREB; 1:500; Abcam, UK), and $\beta$-actin (1:1000; Abcam, UK). Then primary antibodies were removed by washing the membranes three times in TBST. The membranes were further incubated for 2 hours at room temperature with IRDye 680labelled secondary antibodies (1:2000; Santa Cruz). Finally immunoblots were visualized by using enhanced chemiluminescence (ECL; Pierce, Rockford, IL, USA). The optical density of the bands was quantified using ImageJ software (Packard Instruments BV, Groningen, Netherlands). The results were normalized to the quantity of $\beta$-actin in each sample lane. All assays were performed at least three times.

\subsection{Electrophysiological recordings}

NAc slices were prepared from C57BL/6J mice as previously reports with some modifications(M. Li et al., 2018). Briefly, brains were rapidly removed and Sagittal plane brain slices (400 um thickness) containing NAc were cut using a vibrating blade microtome in ice-cold artificial cerebrospinal fluid (ACSF) containing $(\mathrm{mM}) 119 \mathrm{NaCl}, 3.5 \mathrm{KCl}, 1.3 \mathrm{MgSO} 4,2.5 \mathrm{CaCl} 2,1 \mathrm{NaH} 2 \mathrm{PO} 4,26.2 \mathrm{NaHCO} 3$ and 11 glucose that was bubbled continuously with $95 \% \mathrm{O}_{2}-5 \% \mathrm{CO}_{2}$ to adjust $\mathrm{pH}$ to 7.4 . After $2 \mathrm{~h}$ of recovery at $28^{\circ} \mathrm{C}$, an individual slice was transferred to a submerged recording chamber superfused with oxygenated ACSF at $30^{\circ} \mathrm{C}$ at a rate of $3-4 \mathrm{ml} \cdot \mathrm{min}^{-1}$. Prefrontal cortex-accumbal afferents were stimulated by delivering stimuli through a bipolar stimulating electrode implanted into the PFC near the PFC-NAc border $0.5-3 \mathrm{~mm}$ dorsal to the recording electrode placed in the NAc, as we reported(M. Li et al., 2018). Field excitatory postsynaptic potentials (fEPSPs) were recorded by a microelectrode filled with $3 \mathrm{M} \mathrm{NaCl}$ and the test frequency to evoke fEPSPs was $0.03 \mathrm{~Hz}$. Stimulation intensities were chosen to produce a fEPSP with a slope that was $60 \%$ of that obtained with maximal stimulation. Input-output (I/O) relationship for synaptic transmission was recorded by varying the intensity of the single-pulse stimulation. Paired stimuli $(25,50,75,100 \mathrm{~ms}$ interval) were delivered, and the paired-pulse ratio (PPR) was calculated as the ratio between the mean slope of the second fEPSPs over the first fEPSPs. The initial slope of the fEPSPs was measured and expressed as a percentage change from the baseline fEPSPs level, calculated from the average of the last $20 \mathrm{~min}$ of the 
baseline recording period. LTD was induced by the following protocol: NMDAR-dependent LTD in the NAc was induced by a stimulating protocol that consisted of one train of stimulus at $1 \mathrm{~Hz}(15 \mathrm{~min})$ after $10 \mathrm{~min}$ of stable baseline recording. The magnitude of NMDAR-dependent LTD was calculated from fEPSPs recorded after 40 min of LTD induction as the percentage of the baseline EPSP slopes(B. Zhang et al., 2017).

\subsection{Statistical analysis}

All analyses were performed using SPSS 24.0 software (SPSS Inc., USA), and data are presented as mean +- SEM (standard error of the mean). The comparisons between groups were evaluated using one-way ANOVA (post hoc LSD test) or two-way ANOVA (post hoc Bonferroni's test), as appropriate. For all oneway ANOVAs, post hoc tests were performed using LSD test (the least significant difference test). For all two-way ANOVAs, Bonferroni post hoc tests were used to assess isolated comparisons. A value of $\mathrm{P}<0.01$ was considered statistically significant. The data and statistical analysis comply with the recommendations on experimental design and analysis in pharmacology.

\subsection{Materials}

D-serine, K252a and fluoxetine were purchased from Sigma (St. Louis, MO, USA). D-serine and fluoxetine were dissolved in $0.9 \%$ saline. K252a was dissolved in DMSO to $25 \mathrm{mM}$ and in ethanol to $5 \mathrm{mM}$. The dosages of D-serine (2ug/perside,5ug/perside ), fluoxetine (5ug/perside ) and K252a (5ug/perside) were chosen based on previous reports with some modifications(B Jiang et al., 2012). D-serine, K252a and fluoxetine were stereotaxically injected into the bilateral NAc. The repeated drug treatment of mice was performed once daily between 9:30 and 10:30 am.

\section{Results}

\subsection{The concentration of D-serine in NAc was decreased in depression susceptible mice}

After a brief (10 minutes) daily exposure to a highly aggressive resident mouse for 14 days, the mice were divided into control group, depression susceptible group and depression unsusceptible group with 10 mice in each group according to the results of behavioral experiments (data not shown). Then the prefrontal cortex, amygdala and NAc of each group were extracted respectively. Finally, the average content of D-serine in three different brain regions was calculated by high performance liquid chromatography (HPLC). The results showed that there was no significant difference in D-serine content in the prefrontal cortex and amygdala of the control group, depression susceptible group and depression unsusceptible group. However, compared with the control group and the depression unsusceptible group, the content of D-serine in the NAc of depression susceptible group was significantly reduced $(\mathrm{n}=10, \mathrm{P}<0.01$ vs. control or unsusceptible; Figure $1 \mathrm{~A})$. The results showed that the concentration of D-serine in NAc was decreased in depression susceptible group.

\subsection{Antidepressant-like effects of D-serine in the FST and TST of mice}

FST and TST in mice are widely used for detecting potential antidepressant-like activity(Wang et al., 2020). D-serine was injected into the bilateral NAc, with fluoxetine used as the positive control. Analysis indicated that compared with the vehicle group, D-serine $(2,5 \mathrm{ug} /$ perside) or fluoxetine treatment significantly decreased the immobility time in the FST (Figure 2A). Similar to the consequences of FST, D-serine administration robustly reduced the duration of immobility of mice in the TST, compared with the vehicle group (Figure 2B). The magnitude of the D-serine ( $5 \mathrm{ug} /$ perside) induced anti-immobility effect was comparable with that of fluoxetine ( $5 \mathrm{ug} /$ perside).

To exclude the probability that the reduced immobility in these tests might be as a result of an increase in spontaneous locomotor activity(Eissa et al., 2020), mice were exposed to the open-field test. We found no important differences in the number of squares an animal crossed between the center region or the periphery region in all the groups (Figure 2C), and no effects for drug administration. These data indicated that the decrease of immobility observed in the two tests after D-serine disposal was not because of locomotor hyperactivity. Together, the data from the three tests revealed that D-serine may have antidepressant-like effects in control mice. 


\subsection{Chronic D-serine treatment restored the CSDS-induced depressive symptoms}

We further utilized the CSDS model to characterize the antidepressant effects of D-serine, which mimics many symptoms of depression in human(Rouhiainen et al., 2019). As shown in Figure 3A, all mice spent similar time in the interaction zone during the target absent trial. CSDS-defeated mice spent significantly less time in the interaction zone compared to vehicle-treated mice when the CD1 aggressor was present. Interestingly, 14 days of administration with D-serine significantly reversed the CSDS-induced social avoidance behavior, peculiarly at $5 \mathrm{ug} /$ perside, similar to fluoxetine. In addition, CSDS induced a significantly decrease in sucrose preference, compared with control group. 14-day administration with D-serine in CSDS-susceptible mice prompted an obvious increase of sucrose intake (Figure 3B). Collectively, these results revealed that D-serine produces antidepressant effects in depressive mice.

\subsection{Chronic D-serine treatment reverses the CSDS-induced increase BDNF signaling pathway in the NAc}

To investigate the potential mechanisms underlying D-serine-induced antidepressant effects, we inspected the expression of BDNF signaling pathway proteins levels. Data are summarized in Figure 4A. The BDNF expression level was expressed as a ratio of the expression of $\beta$-Actin. As shown in Figure 4, CSDS increased NAc BDNF and pTrkB protein levels compared with the control, which was similar as reported(Dong et al., 2017). Compared with the CSDS group, D-serine treatment significantly decreased their expression. The CREB in the NAc is a significant mediator of neural plasticity and the transcription factor for BDNF. D-serine decreased pCREB level in the NAc of CSDS mice. Thus, D-serine treatment prevented the ability of stress to higher BDNF, pTrkB and pCREB in the NAc. In contrast, the total TrkB and CREB levels were unchanged among all the groups. Moreover, the D-serine-induced decrease in NAc BDNF expression of defeated mice was also improved by K252a treatment $(n=5$; Figure 4). In line with this, K252a also enhanced the effects of D-serine on the expression of NAc pTrkB, and pCREB ( $n=5$; Figure 4). However, the level of total TrkB and CREB protein was not altered. Thus, D-serine-induced changes in the BDNF signaling pathway in the NAc are involved in the mediation of depression.

3.5 D-serine has antidepressant-like effects through restraining the BDNF signaling pathway in the NAc

To determine the relationship between the antidepressant effect of D-serine and the BDNF system, K252a, a potent pharmacological inhibitor of the BDNF receptor TrkB was applied. C57BL/6J mice were premier daily infused with K252a ( $5 \mathrm{ug} /$ perside, injected into the bilateral NAc) for 3 days, then treated with D-serine ( $5 \mathrm{ug} /$ perside), and followed by the FST or TST. It was found that infusion of the K252a alone decreased the immobility of test mice in the FST and TST (Figure 5B and 5C). K252a pretreatment significantly facilitated the antidepressant effects of D-serine in the FST and TST. Furthermore, CSDS-stressed mice were co-treated with D-serine(5ug/perside) and K252a (5ug/perside) for 14 days, and behavioral tests were then implemented. Co-treatment K252a with D-serine significantly enhanced the antidepressant-like effects of D-serine in the sucrose preference test (Figure 5D) and social interaction test (Figure 5E). These results indicate that K252a enhances the antidepressant effects of D-serine.

\subsection{The role of D-serine in synaptic plasticity in the NAc of depressed mice}

\subsubsection{Impaired synaptic plasticity in NAc of depressed mice}

Growing evidence supports the notion that synaptic adjustability in the NAc and its connected circuitry has a principal mission in many forms of reward-dependent learning(Grueter, Robison, Neve, Nestler, \& Malenka, 2013). Furthermore, current studies have certified that synaptic molecular adaptability exist in the neurons of NAc that underlie susceptible and plasticity responses to chronic stress. Three forms of synaptic plasticity at prefrontal cortex-accumbal glutamatergic synapses-paired-pulse facilitation (PPF), input-output relationship(I-O) and LTD - were inspected in sagittal slices by us to identify the adjustment of synaptic plasticity by D-serine.

The PPF is a subtle estimate of the probability of transmitter liberation. Our results revealed that CSDS 
produced no outcomes on PPF, hinting the lack of obvious change in presynaptic action (Figure 6.1A and 6.1B). To determine whether the synaptic effectiveness was varied with chronic stress exposure and antidepressant administration, the input-output(I-O) relationships for field excitatory postsynaptic potentials amplitude were compared. Electrophysiological recordings showed an important reduction in the amplitude of field excitatory postsynaptic potentials in the CSDS group as compared with control group (Figure 6.1C and 6.1D). Moreover, chronic D-serine administration reversed the damage of basic prefrontal cortex-accumbal glutamatergic synapse transmission from CSDS mice (Figure 6.1C and 6.1D). It is suggested that D-serine can improve the basic synaptic damage in CSDS mice. As shown in Figure 6.1 E, F, G, H and I, NMDARdependent LTD of prefrontal cortex-accumbal glutamatergic synapse was impaired in CSDS mice, although it was normal in control mice. It is indicated that depressed mice's synaptic plasticity in NAc was impaired.

\subsubsection{D-Serine in the NAc region modulates Synaptic plasticity}

Furthermore, the NMDAR-LTD in the NAc were compared in the control, CSDS, CSDS+ fluoxetine and CSDS+D-serine(2,5ug/preside) groups. Chronic fluoxetine or D-serine treatment significantly increased the amplitude of LTD and reversed the impaired NMDAR-LTD in NAc of CSDS mice (Figure 6.2 A, B, C and D). Taken together, the electrophysiological data showed that synaptic plasticity in the NAc is disrupted in CSDS mice, which was reversed by D-serine treatment, backing a role for NMDAR-LTD in the NAc in depression.

\section{Discussion}

The crucial discoveries of this research are as follows. Initially, the concentration of D-serine was decreased in depression susceptible mice in NAc. Secondly, D-serine produced antidepressant-like effects in the FST, TST and CSDS model. Thirdly, chronic treatment of D-serine could restore the stress-induced increase in NAc BDNF signaling cascade. Next, reduced levels of D-Serine in the NAc contribute to impaired synaptic plasticity. Together, we have demonstrated for the first time that D-serine has beneficial effects through suppression of the BDNF signaling pathway and regulation of synaptic adaptations in NAc against depression. This discovery is extremely exciting and interesting as it has provided an original underlying antidepressant.

D-serine can pass through the blood-brain barrier, and we have simultaneously completed the in vivo experiment of intraperitoneal injection. Our data showed that D-serine has capabilities common to traditional antidepressants in the FST and TST. In these tests, mice were under stressful circumstances from which they could not escape, and would become helpless and immobile after a premier period of striving, which is similar to human depression and could be reversed by antidepressant pharmaceuticals. As expected, the reduction of immobility in the FST and TST induced by acute injection of D-serine in mice was not codirectional by an increase in locomotor activity of mice, indicating that D-serine indeed has a potential interest for the treatment of depression. We further used the CSDS model to make sure the outcomes of D-serine, as the CSDS model has great predictive validity the symptomatology of stress-related disorders like depression(K. Zhang et al., 2020). The sucrose preference test is an indicatrix of the anhedonia-like behavioral alterations. Our consequences demonstrated that D-serine administration fully renovated the sucrose preference of CSDS-stressed mice to normal level. Another marked symptom of depression is social elusion, meaning that depressed rodents are passive and tempting to get away from the world. Our results bespoken that Dserine administration also reversed the social avoidance of CSDS-stressed mice, like fluoxetine. Collectively, these discoveries demonstrate that D-serine could be developed into a novel antidepressant.

Moreover, the discovery that D-serine could reverse the CSDS-induced effects on the NAc BDNF signaling cascade is infusive. This research is the premier to provide experimental evidence representing that D-serine has effects on the BDNF system in NAc. In this research, we found that chronic administration of D-serine down-regulated level of BDNF, phosphorylated TrkB and phosphorylated CREB in the NAc of stressed mice, to the basal level of saline-treated mice. Moreover, the antidepressant effects induced by D-serine administration were also facilitated by co-treatment with K252a, a potent inhibitor of the BDNF receptor TrkB. 
Synaptic plasticity is regarded as a cellular substrate of learning and memory(Kamalova, Futai, Delpire, \& Nakagawa, 2020). D-Serine is considerable for the activation of NMDAR-dependent synaptic plasticity(Cha, Lee, \& Lee, 2020). NAC neurons are among those that lightly express LTD, which is susceptible to stress. We found that D-serine facilitated LTD. It has been confirmed that D-Serine in NAc modulates drug addiction through synaptic plasticity(Liu et al., 2016). It is well known that drug addiction and depression are regulated by NAc and are two opposite behaviors. Our results support that LTD was markedly damaged in the NAc of CSDS mice and was reversed by a chronic injection of D-serine. LTD is induced by low rises of the intracellular $\mathrm{Ca}^{2+}$ concentration(Bezprozvanny \& Hiesinger, 2013). Reduced $\mathrm{Ca}^{2+}$ influx at postsynaptic level with hypofunction of NMDAR could persuasively explain the damage of LTD in our CSDS model. So, our results manifested that the decrease of D-serine levels in the NAc of CSDS mice hindered the induction of NMDAR-dependent LTD. By electrophysiological techniques, our consequences sustain the NMDAR-LTD molecular substrate that convey this lack of plasticity in CSDS and could work out new targets for antidepressants.

In summary, the results of this study reveal that D-serine possesses antidepressant-like effects in mice, which appeared to be mediated through down-regulation of the NAc BDNF-TrkB signaling pathway. In addition, it indicates that chronic social defeat stress treatment can alter the metabolism of D-serine in the NAc, leading to both reduced D-serine levels and the impairment of NMDAR dependent LTD. Moreover, increasing the content of D-serine in NAC can improve depression. Therefore, this provides a new insight to see the pharmacological outcomes of D-serine and illuminating the development of new antidepressants with higher effectiveness and fewer side reaction. Furthermore, related experiments such as SR-shRNA, DAAO-shRNA, NMDAR blocker MK-801, NR2B-shRNA and so on by us are in progress at the same time.

\section{ACKNOWLEDGEMENTS}

This work was supported by grants from the National Natural Science Funds of China (Grant No. 81760256 and 81960256) to Dr. Wei Wang. The study also received support by Academic and technical leaders of major disciplines Foundation (20204BCJL22049) and Natural Science Foundation (Grant No. 20192BAB205038) of Jiangxi, China to Dr. Wei Wang.

\section{AUTHOR CONTRIBUTIONS}

Wei Wang and Zhenzhen Chen conceived and designed this study. Zhenzhen Chen, Ke Zou, Zhihong Huang and Liuer Liu performed the experiments. Zhenzhen Chen wrote the manuscript. Zhenyu Tang, Yuanjian Yang and Wei Wang reviewed and edited this manuscript. All the authors read and approved this manuscript.

\section{CONFLICT OF INTEREST}

The authors declare no conflicts of interest.

\section{DECLARATION OF TRANSPARENCY AND SCIENTIFIC RIGOUR}

This Declaration acknowledges that this paper adheres to the principles for transparent reporting and scientific rigor of preclinical research recommended by funding agencies, publishers and other organizations engaged with supporting research.

\section{DATA AVAILABILITY STATEMENT}

The data that support the findings of this study are available from the corresponding author upon reasonable request. Some data may not be made available because of privacy or ethical restrictions.

\section{ORCID}

Zhenzhen Chenhttps://orcid.org/0000-0002-0765-5574

\section{REFERENCES}

Alhusaini, A., Hasan, I., Alrumayyan, B., Alesikri, M., Alanazi, K., Almasoud, R., \& Almarshad, S. (2020). Neuroprotective efficacy of nano-CoQ against propionic acid toxicity in rats: Role of BDNF and CREB 
protein expressions. Journal of biochemical and molecular toxicology, 34 (4), e22449. doi:10.1002/jbt.22449

Arora, S., Sharma, D., \& Singh, J. (2020). GLUT-1: An Effective Target To Deliver Brain-Derived Neurotrophic Factor Gene Across the Blood Brain Barrier. ACS chemical neuroscience, 11 (11), 1620-1633. doi:10.1021/acschemneuro.0c00076

Bai, L., Zhang, S., Zhou, X., Li, Y., \& Bai, J. (2019). Brain-derived neurotrophic factor induces thioredoxin-1 expression through TrkB/Akt/CREB pathway in SH-SY5Y cells. Biochimie, 160 , 55-60. doi:10.1016/j.biochi.2019.02.011

Belujon, P., \& Grace, A. (2014). Restoring mood balance in depression: ketamine reverses deficit in dopamine-dependent synaptic plasticity.Biol Psychiatry, 76 (12), 927-936. doi:10.1016/j.biopsych.2014.04.014

Bezprozvanny, I., \& Hiesinger, P. (2013). The synaptic maintenance problem: membrane recycling, Ca2+ homeostasis and late onset degeneration. Molecular neurodegeneration, 8 , 23. doi:10.1186/1750-1326-8-23

Caroleo, M., Carbone, E., Primerano, A., Foti, D., Brunetti, A., \& Segura-Garcia, C. (2019). The role of hormonal, metabolic and inflammatory biomarkers on sleep and appetite in drug free patients with major depression: A systematic review. Journal of affective disorders, 250 , 249-259. doi:10.1016/j.jad.2019.03.015

Cha, M., Lee, K., \& Lee, B. (2020). Astroglial changes in the zona incerta in response to motor cortex stimulation in a rat model of chronic neuropathy. Scientific reports, 10 (1), 943. doi:10.1038/s41598-020$57797-\mathrm{y}$

Dong, C., Zhang, J., Yao, W., Ren, Q., Ma, M., Yang, C., ... Hashimoto, K. (2017). Rapid and Sustained Antidepressant Action of the mGlu2/3 Receptor Antagonist MGS0039 in the Social Defeat Stress Model: Comparison with Ketamine. Int J Neuropsychopharmacol, 20 (3), 228-236. doi:10.1093/ijnp/pyw089

Eissa, N., Azimullah, S., Jayaprakash, P., Jayaraj, R., Reiner, D., Ojha, S., . . Sadek, B. (2020). The DualActive Histamine H Receptor Antagonist and Acetylcholine Esterase Inhibitor E100 Alleviates Autistic-Like Behaviors and Oxidative Stress in Valproic Acid Induced Autism in Mice. International journal of molecular sciences, 21 (11)doi:10.3390/ijms21113996

Grueter, B., Robison, A., Neve, R., Nestler, E., \& Malenka, R. (2013). [?]FosB differentially modulates nucleus accumbens direct and indirect pathway function. Proceedings of the National Academy of Sciences of the United States of America, 110 (5), 1923-1928. doi:10.1073/pnas.1221742110

He, Z., Fan, R., Zhang, C., Tang, T., Liu, X., Luo, J., ... Gan, P. (2019). Chaihu-Shugan-San Reinforces CYP3A4 Expression via Pregnane X Receptor in Depressive Treatment of Liver-Qi Stagnation Syndrome.Evidence-based complementary and alternative medicine : eCAM, 2019 , 9781675. doi:10.1155/2019/9781675

Jiang, B., Wang, F., Yang, S., Fang, P., Deng, Z. F., Xiao, J. L., .. Chen, J. G. (2014). SKF83959 produces antidepressant effects in a chronic social defeat stress model of depression through BDNF-TrkB pathway. Int J Neuropsychopharmacol, 18 (6)doi:10.1093/ijnp/pyu096

Jiang, B., Wang, W., Wang, F., Hu, Z., Xiao, J., Yang, S., ... Chen, J. (2013). The stability of NR2B in the nucleus accumbens controls behavioral and synaptic adaptations to chronic stress. Biol Psychiatry, 74 (2), 145-155. doi:10.1016/j.biopsych.2012.10.031

Jiang, B., Xiong, Z., Yang, J., Wang, W., Wang, Y., Hu, Z., .. Chen, J. (2012). Antidepressant-like effects of ginsenoside Rg1 are due to activation of the BDNF signalling pathway and neurogenesis in the hippocampus. Br J Pharmacol, 166 (6), 1872-1887. doi:10.1111/j.1476-5381.2012.01902.x

Jiang, X., Lin, W., Cheng, Y., \& Wang, D. (2020). mGluR5 Facilitates Long-Term Synaptic Depression in a Stress-Induced Depressive Mouse Model. Canadian journal of psychiatry. Revue canadienne de psychiatrie, 65 (5), 347-355. doi:10.1177/0706743719874162 
Jin, Y., Cui, R., Zhao, L., Fan, J., \& Li, B. (2019). Mechanisms of Panax ginseng action as an antidepressant. Cell proliferation, 52 (6), e12696. doi:10.1111/cpr.12696

Kamalova, A., Futai, K., Delpire, E., \& Nakagawa, T. (2020). AMPA Receptor Auxiliary Subunit GSG1L Suppresses Short-Term Facilitation in Corticothalamic Synapses and Determines Seizure Susceptibility.Cell reports, 32 (3), 107921. doi:10.1016/j.celrep.2020.107921

Kowiański, P., Lietzau, G., Czuba, E., Waśkow, M., Steliga, A., \& Moryś, J. (2018). BDNF: A Key Factor with Multipotent Impact on Brain Signaling and Synaptic Plasticity. Cellular and molecular neurobiology, 38 (3), 579-593. doi:10.1007/s10571-017-0510-4

Li-Wen, He, Li, Zeng, Na, Tian, ... He. (2020). Optimization of food deprivation and sucrose preference test in SD rat model undergoing chronic unpredictable mild stress. Animal Models and Experimental Medicine, v.3 (01), 73-82.

Li, D., Wang, Y., Jin, X., Hu, D., Xia, C., Xu, H., \& Hu, J. (2020). NK cell-derived exosomes carry miR-207 and alleviate depression-like symptoms in mice. Journal of neuroinflammation, 17 (1), 126. doi:10.1186/s12974-020-01787-4

Li, M., Zheng, H., Luo, Y., He, J., Wang, W., Han, J., .. Wang, F. (2018). Gene deficiency and pharmacological inhibition of caspase- 1 confers resilience to chronic social defeat stress via regulating the stability of surface AMPARs. Molecular psychiatry, 23 (3), 556-568. doi:10.1038/mp.2017.76

Liu, Z., Gu, X., Yang, Y., Yin, X., Xu, L., \& Wang, W. (2016). D-Serine in the nucleus accumbens region modulates behavioral sensitization and extinction of conditioned place preference. Pharmacol Biochem Behav, 143 , 44-56. doi:10.1016/j.pbb.2016.02.002

Lorsch, Z., Loh, Y., Purushothaman, I., Walker, D., Parise, E., Salery, M., .. Nestler, E. (2018). Estrogen receptor $\alpha$ drives pro-resilient transcription in mouse models of depression. Nature communications, 9 (1), 1116. doi:10.1038/s41467-018-03567-4

Ntoukas, A., Niarchos, A., Tsika, A., Mantzoukas, S., Spyroulias, G., \& Poulas, K. (2020). A quantitative western blot technique using TMB: Comparison with the conventional technique.Electrophoresis doi:10.1002/elps.202000306

Rouhiainen, A., Kulesskaya, N., Mennesson, M., Misiewicz, Z., Sipilä, T., Sokolowska, E., ... Hovatta, I. (2019). The bradykinin system in stress and anxiety in humans and mice. Scientific reports, 9 (1), 19437. doi:10.1038/s41598-019-55947-5

Shirayama, Y., \& Chaki, S. (2006). Neurochemistry of the nucleus accumbens and its relevance to depression and antidepressant action in rodents. Current neuropharmacology, 4 (4), 277-291. doi:10.2174/157015906778520773

Snyder, S. H., \& Kim, P. M. J. N. R. (2000). D-amino acids as putative neurotransmitters: focus on D-serine. $25(5), 553-560$.

Taylor, S., \& Posch, A. (2014). The design of a quantitative western blot experiment. Biomed Res Int, 2014 , 361590. doi:10.1155/2014/361590

Tomassoni-Ardori, F., Fulgenzi, G., Becker, J., Barrick, C., Palko, M., Kuhn, S., ... Tessarollo, L. (2019). Rbfox1 up-regulation impairs BDNF-dependent hippocampal LTP by dysregulating TrkB isoform expression levels. eLife, 8 doi:10.7554/eLife.49673

Wang, C., Gong, S., Guan, W., Wang, J., Gao, T., Wang, Y., ... Jiang, B. (2020). Hippocampal overexpression of chordin protects against the chronic social defeat stress-induced depressive-like effects in mice. Brain research bulletin, 158 , 31-39. doi:10.1016/j.brainresbull.2020.02.010

Wook Koo, J., Labonté, B., Engmann, O., Calipari, E., Juarez, B., Lorsch, Z., .. Nestler, E. (2016). Essential Role of Mesolimbic Brain-Derived Neurotrophic Factor in Chronic Social Stress-Induced Depressive 
Behaviors. Biol Psychiatry, 80 (6), 469-478. doi:10.1016/j.biopsych.2015.12.009

Xu, D., Wang, C., Zhao, W., Gao, S., \& Cui, Z. (2017). Antidepressant-like effects of ginsenoside Rg5 in mice: Involving of hippocampus BDNF signaling pathway. Neurosci Lett, 645 , 97-105. doi:10.1016/j.neulet.2017.02.071

Zhang, B., Wang, L., Chen, T., Hong, J., Sha, S., Wang, J., .. Chen, L. (2017). Sigma-1 receptor deficiency reduces GABAergic inhibition in the basolateral amygdala leading to LTD impairment and depressive-like behaviors. Neuropharmacology, 116 , 387-398. doi:10.1016/j.neuropharm.2017.01.014

Zhang, K., Lin, W., Zhang, J., Zhao, Y., Wang, X., \& Zhao, M. (2020). Effect of Toll-like receptor 4 on depressive-like behaviors induced by chronic social defeat stress. Brain and behavior, 10 (3), e01525. doi:10.1002/brb3.1525

Zhi, Y., Jin, Y., Pan, L., Zhang, A., \& Liu, F. (2019). Schisandrin A ameliorates MPTP-induced Parkinson's disease in a mouse model via regulation of brain autophagy. Archives of pharmacal research, 42 (11), 10121020. doi:10.1007/s12272-019-01186-1

Zhong, W., Yuan, Y., Gu, X., Kim, S., Chin, R., Loye, M., ... Yu, S. (2020). Neuropsychological Deficits Chronically Developed after Focal Ischemic Stroke and Beneficial Effects of Pharmacological Hypothermia in the Mouse. Aging and disease, 11 (1), 1-16. doi:10.14336/ad.2019.0507

\section{Hosted file}

Figure.pdf available at https://authorea.com/users/404327/articles/515548-d-serine-hasantidepressant-effects-in-mice-through-suppression-of-the-bdnf-signaling-pathway-andregulation-of-synaptic-plasticity-in-the-nucleus-accumbens 